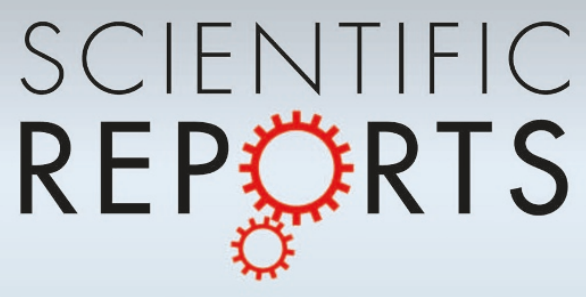

\title{
OPEN Modified Li chains as atomic switches
}

\section{SUBJECT AREAS: CONDENSED-MATTER PHYSICS \\ APPLIED PHYSICS \\ NANOSCIENCE AND \\ TECHNOLOGY}

THEORY AND COMPUTATION

Received

21 June 2013

Accepted

22 August 2013

Published

6 September 2013

Correspondence and requests for materials should be addressed to

U.S. (udo.

schwingenschlogl@

kaust.edu.sa)

\author{
Thomas Wunderlich', Berna Akgenc',2, Ulrich Eckern', Cosima Schuster' \& Udo Schwingenschlögl ${ }^{3}$
}

${ }^{1}$ Institut für Physik, Universität Augsburg, 86135 Augsburg, Germany, ${ }^{2}$ Kirklareli University, Physics Department, Kavakli, Kirklareli, Turkey, ${ }^{3}$ KAUST, PSE Division, Thuwal 23955-6900, Kingdom of Saudi Arabia.

We present electronic structure and transport calculations for hydrogen and lithium chains, using density functional theory and scattering theory on the Green's function level, to systematically study impurity effects on the transmission coefficient. To this end we address various impurity configurations. Tight-binding results allow us to interpret our the findings. We analyze under which circumstances impurities lead to level splitting and/or can be used to switch between metallic and insulating states. We also address the effects of strongly electronegative impurities.

E lectronic devices have been reduced more and more in size over the last decades. Furthermore it is now possible to place atoms or molecules accurately between macroscopic electrodes, hence experimental studies of the electronic transport for single atoms ${ }^{1,2}$, molecules ${ }^{3}$, and nanowires ${ }^{4}$ have become available. One-dimensional structures are of particular interest due to their restricted transport channels, making them prototypical model systems. For example, mono-atomic chains have been realized by molecular beam epitaxy ${ }^{1}$. Using the tip of a scanning tunneling microscope, it has been possible to place a row of eight atoms on a NiAl substrate ${ }^{5}$.

From the theoretical point of view, transport through distorted one-dimensional systems has been addressed already in the early 1990ies by bosonization techniques ${ }^{6-8}$. Transport properties of lattice models are currently investigated on several levels. Comparison of an exact treatment by the density matrix renormalization group ${ }^{9}$ with approaches using density functional theory ${ }^{10}$ shows that the latter approach often is sufficient to calculate the linear conductance, at least qualitatively. The generalization of density functional theory to time-dependent potentials ${ }^{11}$ allows to study the propagation in time of the electronic states ${ }^{12}$. To model the experimental setup more realistically, several methods have been developed for describing the transmission through nano-contacts. Tight-binding formulations have been applied to metallic nano-contacts ${ }^{13,14}$, adding orbital information from chemical analysis; however, these may fail in the contact regime. Nowadays, most approaches rely on a combination of density functional theory and a scattering approach on the non-equilibrium Green's function level, based on the Landauer-Büttiker scheme ${ }^{15}$. In this article, the electronic structure and the transmission coefficient are determined for $\mathrm{H}$ and $\mathrm{Li}$ chains with defects.

Of course, experimental as well as theoretical studies of (charge and spin) transport through nanoscopic structures are numerous-with the technologically relevant goal to develop devices where the transport properties can be switched in a controlled way; see, e.g. Refs. 16,17. With our study, even though we concentrate on simple model systems (cf. Ref. 18), we add to these efforts by giving a systematic investigation of how impurities modify the transmission coefficient, and how these modifications depend on the details of the configurations. It will become clear from the discussions given below that our results provide important insights of a more general nature.

\section{Results}

The impurity models studied in the following are displayed in Fig. 1. The parent structure is a finite (metallic) Li chain with three atoms, which is coupled to $\mathrm{H}$-chain leads. By varying the distance between the last $\mathrm{H}$ and the first Li atom, we change the coupling from strong $(a=2.8 \AA)$ to weak $(a=4.0 \AA)$. The distance between the Li atoms within the chain is fixed to $b=3 \AA$. These values are chosen such as to allow easy comparison with the results presented in Ref. 18.

The impurities are Li atoms adjacent to the parent chain, thus breaking the rotational and/or inversion symmetry of the system. In configuration A an additional Li atom is placed directly below a chain atom, cf. Ref. 19. The distance $d$ between the ad-atom and the chain is varied. A second atom on the other side of the chain, configuration B, restores the symmetry. An additional atom can also be placed below a Li-Li bond, configuration $\mathrm{C}$, which also breaks the symmetry in the transport direction. In addition, we study configuration D in which we place the ad-atom below the central bond, to obtain a system with inversion symmetry. 


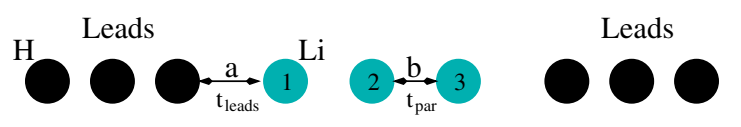

(a)

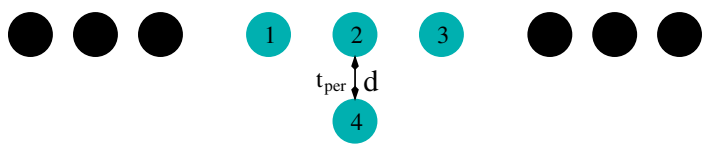

(b)
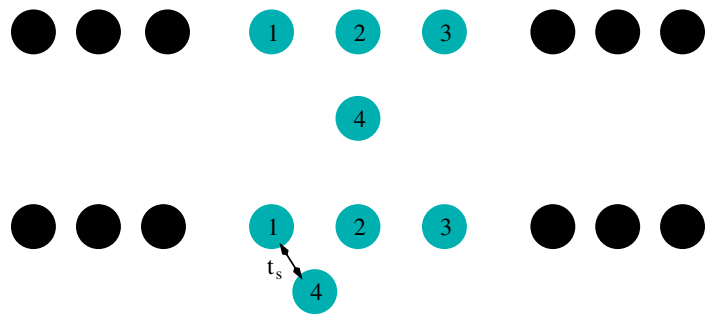

(c)

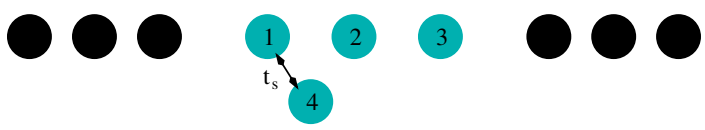

(d)

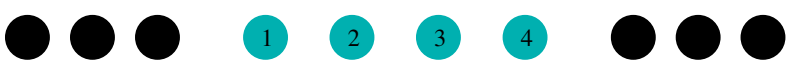

As the transmission coefficient shows resonance peaks at the energy levels of the scattering region, an analysis of this spectrum allows an explanation of the behavior of $T(E)$. For this purpose, the energy levels can be discussed on the basis of a tight-binding approximation with nearest-neighbor hopping. With the hopping parameters indicated in Fig. 1, the Hamiltonian for a three-atom Li chain is given by

$$
H=-t_{\mathrm{par}}\left(c_{1}^{+} c_{2}+c_{2}^{+} c_{3}+\text { h.c. }\right),
$$

where the sites are numbered 1,2 , and 3 . The hopping energy along the chain, $t_{\mathrm{par}}$, is of the order of $2 \mathrm{eV}$, as the bandwidth of a Li chain with atomic spacing $b=3 \AA$ is $\sim 4.5 \mathrm{eV}$. The three-atom chain has the energy levels $\varepsilon_{1,3}= \pm \sqrt{2} t_{\mathrm{par}}$, and $\varepsilon_{2}=0$. The coupling to the leads can be taken into account by an additional hopping to the left and to the right of the scattering region. The hopping to the ad-atoms is denoted by $t_{\mathrm{per}}$, thus $H_{\mathrm{per}}=-t_{\mathrm{per}}\left(c_{2}^{+} c_{4}+\right.$ h.c. $)$ for configuration $\mathrm{A}$, and $H_{\mathrm{per}}=-t_{\mathrm{per}}\left(c_{2}^{+} c_{4}+c_{2}^{+} c_{5}+\right.$ h.c. $)$ for B. In case of configurations $\mathrm{C}$ and $\mathrm{D}$, the hopping terms are given by $-t_{\mathrm{s}}\left(c_{1}^{+} c_{4}+\right.$ $c_{2}^{+} c_{4}+$ h.c. $)$, and $-t_{\mathrm{s}}\left(c_{2}^{+} c_{5}+c_{3}^{+} c_{5}+\right.$ h.c. $)$, respectively. We start our discussion with a review of results for $\mathrm{H}$ chains, which is the simplest case of one-dimensional scatterers, see the structural setup in Fig. 2. Ke and coworkers ${ }^{18}$ have used a system of infinite $\mathrm{H}$ chains as leads and a finite six-atom $\mathrm{H}$ chain for their transport calculations, focusing on the influence of the exchange-correlation potential and the according self-interaction errors. In the following we use the local density approximation, because of its simplicity, and since it reproduces reasonably well the qualitative behavior of the transmission coefficient ${ }^{10}$. Clearly, $T(E)$ depends on the energy levels of the central $\mathrm{H}$ chain, and on the coupling strengths to the leads. The number of atoms within the chain determines the number of energy levels, and hence the number of resonances, see Fig. 2. A five-atom chain

is found to be metallic, whereas a six-atom chain turns out to be

Figure $1 \mid$ Ad-atom configurations under consideration. The distance $d$ between the ad-atom(s) and the chain is varied. The $t$-parameters refer to the tight-binding description of the central scattering region; see main text. The impurity configurations studied, (a)-(d), are denoted A-D, respectively, in the main text for clarity.
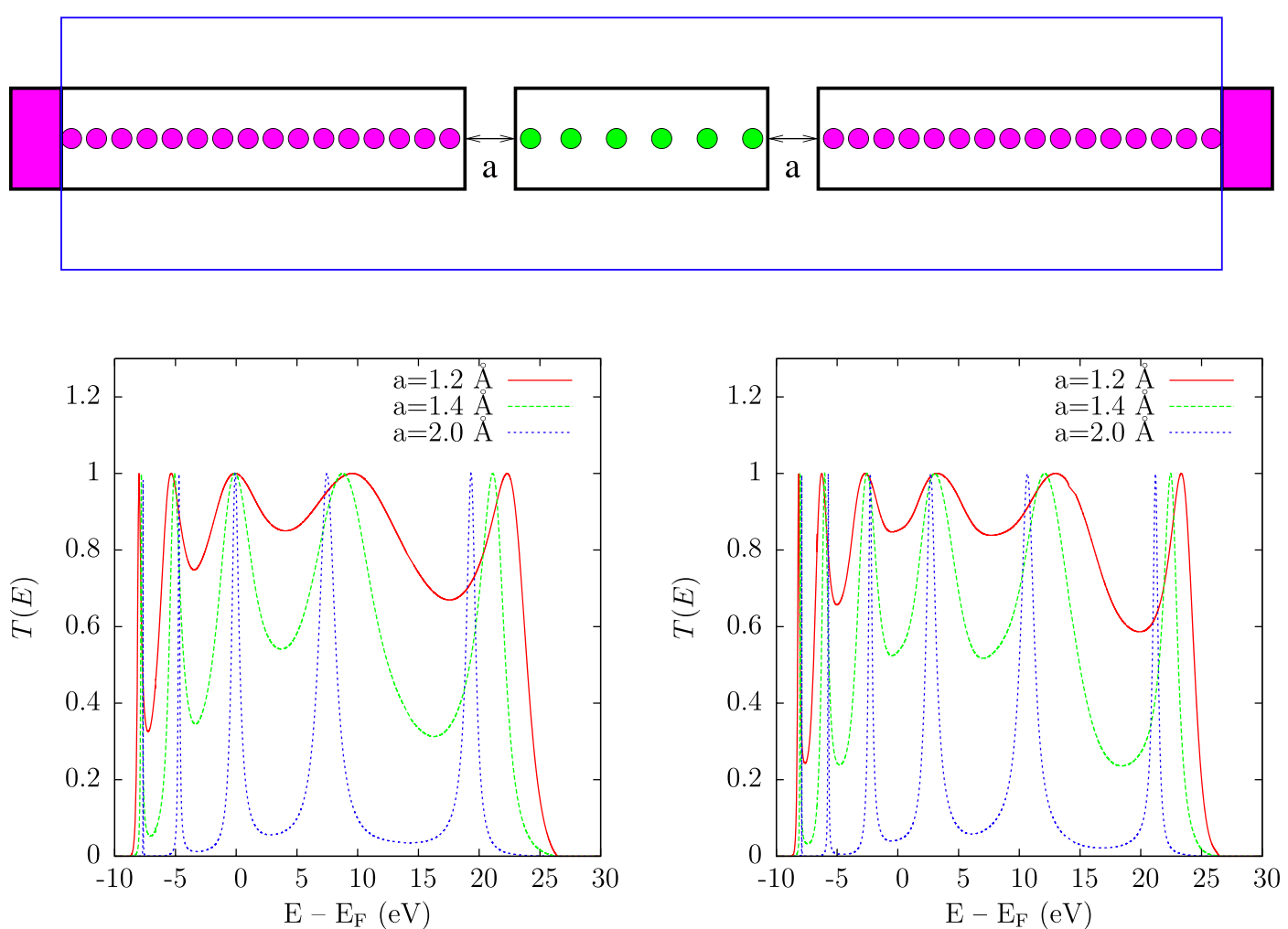

Figure $2 \mid$ Transmission coefficients of five-atom (left) and six-atom (right) $\mathrm{H}$ chains coupled to $\mathrm{H}$ leads, upon increasing the distance a between the scattering region and the leads. The bond lengths in the scattering region and in the leads are fixed at $1.0 \AA$ A. 
insulating. This odd-even behavior is reflected by a maximum or minimum in $T\left(E_{F}\right)$ in Fig. 2. Moreover, the transmission peaks become broader with increasing coupling to the leads, and also shift in energy.

Considering in addition the bond length within the chain, $b$, as a parameter, we find, as expected, a decrease of the band width with increasing $b$. For $b=1.0 \AA$ the bands are close to parabolic, and the unoccupied levels are more sensitive when decreasing the coupling than the occupied levels. With increasing $b$, the dispersion becomes more and more symmetric and cosine shaped, consistent with the tight-binding description. Keeping the lead-chain distance fixed at, say, $a=1.2 \AA$ but increasing the bond length $b$ beyond this value, one arrives at a situation where the first and the last atom of the central region are effectively bound to the leads, i.e., the scattering region is a diluted chain, however, with two atoms less. Note that we use the terms "central region", "scattering region", and "scatterer" synonymously.

When a finite Li chain is coupled to $\mathrm{H}$ chain leads, charge is transfered between the leads and the scattering region. For an even number of Li atoms this charge transfer results in a level shift towards $E_{F}{ }^{18}$. Extending the studies of ${ }^{18}$, we discuss in the following the influence of perturbations.

(a)

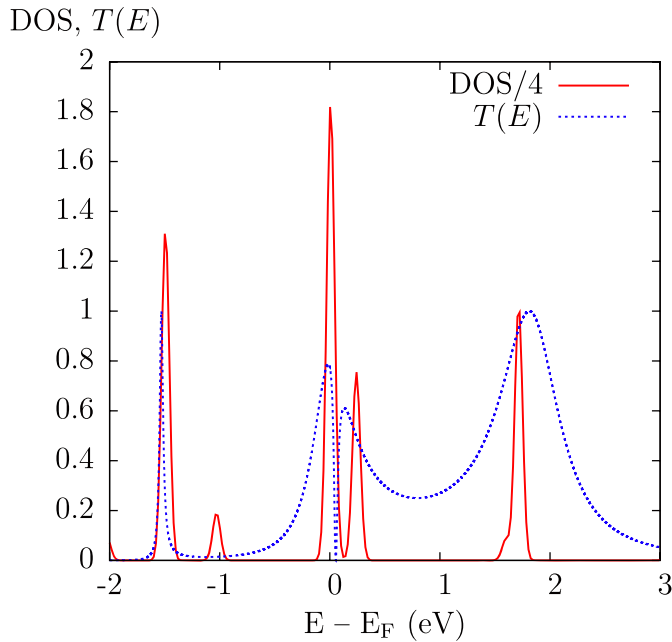

(c)

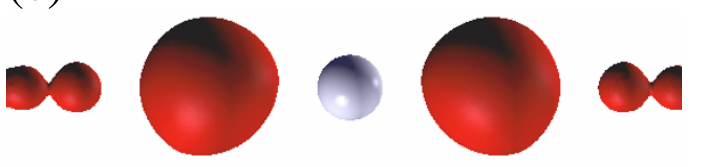

$$
\text { HOMO }
$$

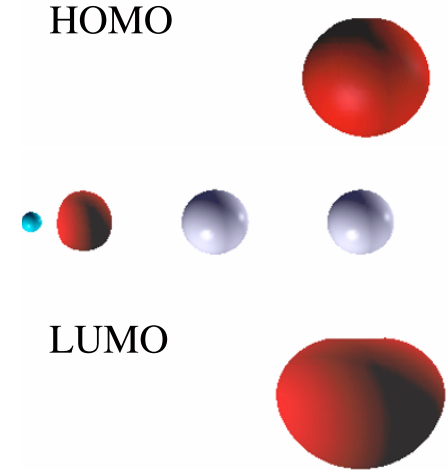

For configuration $\mathrm{A}$, with an additional $\mathrm{Li}$ atom adjacent to the $\mathrm{Li}$ chain, the tight-binding energy levels of the scatterer are given by $\varepsilon_{1,4}= \pm \sqrt{2 t_{\mathrm{par}}^{2}+t_{\mathrm{per}}^{2}}$, and $\varepsilon_{2,3}=0$. The coupling to the leads lifts the degeneracy of the zero-energy level. A comparison of the density of states (DOS) obtained by SIESTA with $T(E)$ is given in Fig. 3(a) for a projection of the DOS onto the Li atoms. The distance of the ad-atom to the chain is $d=3.5 \AA$. The four energy levels of the scatterer are located at $-1.5 \mathrm{eV}, 0 \mathrm{eV}=E_{F}, 0.3 \mathrm{eV}$, and $1.7 \mathrm{eV}$. The smaller peak at $-1.0 \mathrm{eV}$ as well as the shoulder at $1.6 \mathrm{eV}$ arise from hybridization with atoms in the leads. When the distance between the ad-atom and the $\mathrm{Li}$ chain is increased, the splitting of the states near $E_{F}$ becomes more pronounced, see Fig. 3(b).

Analyzing the spatial distribution of the charge for each energy level yields strong similarities between the tight-binding model and the DFT calculation. The lowest level corresponds to a homogeneous charge distribution over the scatterer. The second level (highest occupied molecular orbital, HOMO) resides on Li atoms 1, 3, and 4, whereas the third level (lowest unoccupied molecular orbital, LUMO) is dominated by the ad-atom, see Fig. 3(c). In the range $d$ $=3.5 \ldots 4.0 \AA$ a redistribution of weight from the HOMO to the LUMO is apparent. For increasing distance $d$, the charge becomes more localized on the ad-atom, resulting in a stronger transmission

(b)

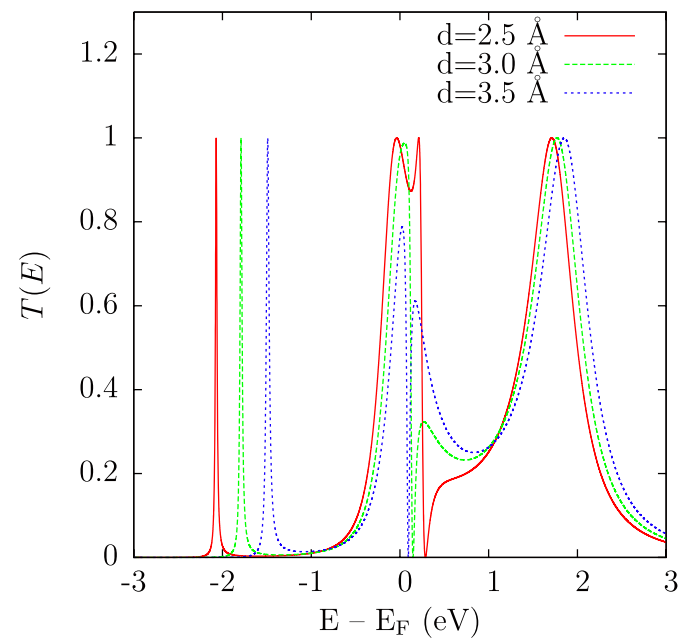

(d)

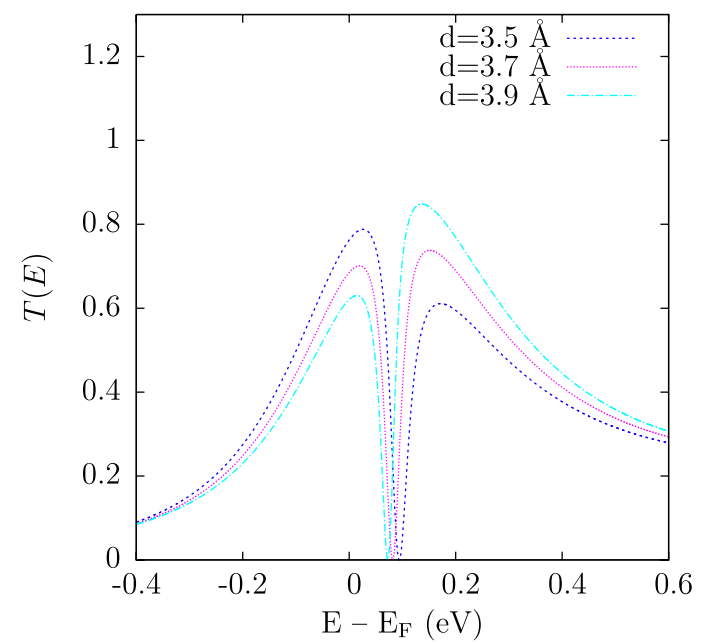

Figure $3 \mid$ (a) DOS (divided by 4, for easy comparison, in units of $1 / \mathrm{eV}$ ) and transmission coefficient of configuration A for $d=3.5 \AA$. (b) Transmission coefficient for various distances between chain and impurity. (c) Charge density iso-surfaces of the HOMO and the LUMO. (d) Zoom of (b) at $E_{F}$. 

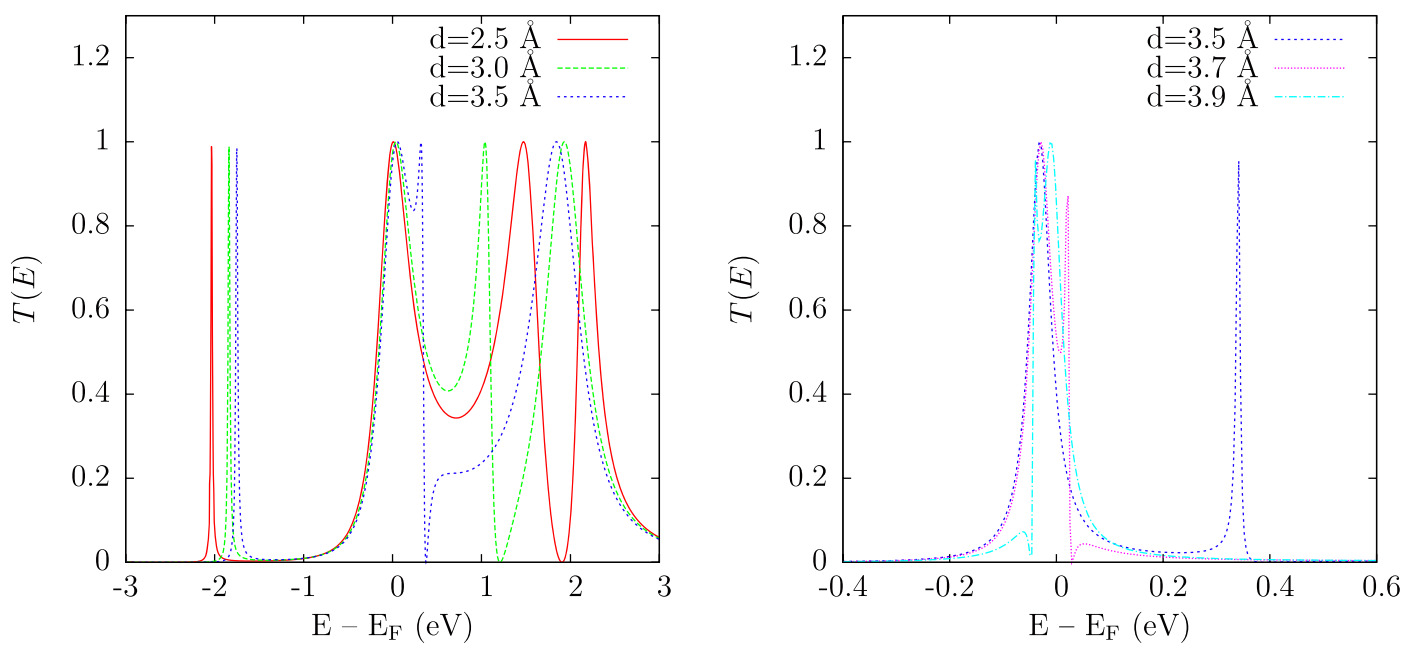

Figure $4 \mid$ Transmission coefficient for configuration B with strong $(a=2.8 \AA$, left $)$ and weak $(a=4.0 \AA$, right $)$ coupling to the leads.

through the LUMO. The minimum of the total energy appears for $d$ $=2.75 \AA$; then the energy increases almost linearly when increasing $d$ (up to $d=4.0 \AA$ ).

For the symmetrical configuration B, see Fig. 4, we find five energy levels in the tight-binding spectrum: bonding and antibonding levels $\varepsilon_{1,5}= \pm \sqrt{2 t_{\text {par }}^{2}+2 t_{\text {per }}^{2}}$ as well as $\varepsilon_{2,3,4}=0$. A next-nearest-neighbor hopping $t_{\mathrm{nn}}$ shifts one of the zero-energy levels, say, $\varepsilon_{4}$, to a finite value, given by $\varepsilon_{4} \approx 2 t_{\mathrm{nn}} t_{\text {per }} / t_{\text {par }}$ (which holds in the limit $t_{\mathrm{nn}}, t_{\mathrm{per}} \ll$ $\left.t_{\text {par }}\right)$. The coupling to the leads also lifts the threefold degeneracy of $\varepsilon_{2,3,4}$. As a result, a spectrum of five levels is obtained, with two nearly degenerate levels near the Fermi energy, see Fig. 4. Level 4, corresponding to $\varepsilon_{4}$, has a rather homogeneous charge distribution over the scattering region for a small distance, $d=2.5 \AA$. With increasing distance, the contribution of atom 2 to the charge density decreases, and hence the transmission through this level.

The position of the fourth peak in $T(E)$ depends strongly on the distance between the ad-atoms and the chain. For $d=3.5 \AA$, for example, it is located at about $0.5 \mathrm{eV}$, whereas it is found at $1.0 \mathrm{eV}$ for $d=3.0 \AA$ (equilibrium distance). The two peaks near $E_{F}$ cannot be resolved on the left hand side of Fig. 4 due to the coupling to the leads. For weak coupling a three-peak structure around $E_{F}$ is resolved, again showing the redistribution of weight below and above $E_{F}$, compare Fig. 3(d) with the right hand side of Fig. 4. The sharp features

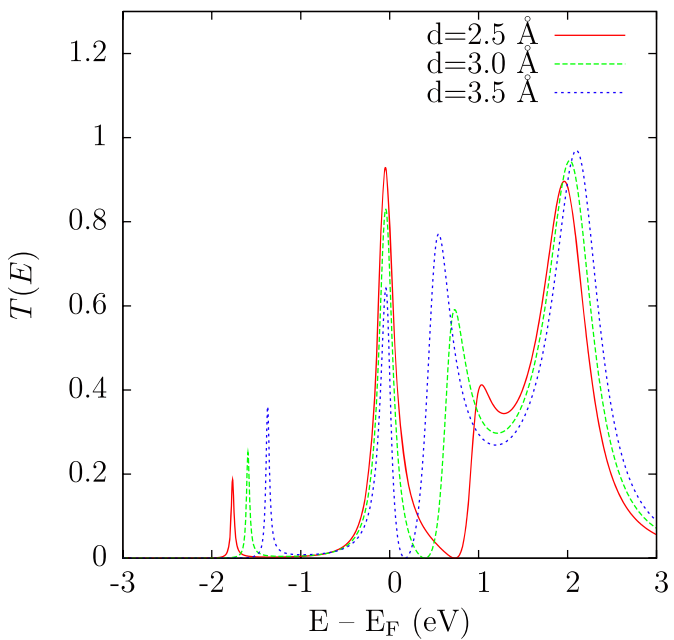

observed near the Fermi energy in Figs. 3 and 4 are very similar to previous results ${ }^{20-22}$. As discussed in Refs. 20-22, these features are most likely a Fano resonance.

Configuration $\mathrm{C}$ corresponds to a scatterer which is asymmetric in both in-plane directions. The tight-binding model yields the spectrum $\varepsilon_{1,4} \approx \pm \sqrt{2} t_{\text {par }}$ and $\varepsilon_{2,3} \approx \pm t_{\mathrm{s}} / \sqrt{2}$ provided $t_{\mathrm{s}}$ is small, $t_{\mathrm{s}} \ll t_{\mathrm{par}}$, i.e., when the distance between ad-atom and chain is large. The first guess thus is that the system should be insulating, in contrast to the parent chain and to the configuration where the ad-atom is attached to a single chain atom. According to Fig. 5, however, $T(E)$ does not show a minimum at $E_{F}$ : in fact, due to the charge transfer with the leads the HOMO is fixed near $E_{F}$, as demonstrated in Ref. 18 (also within DFT). In addition, the lowest level shifts slightly in energy when varying $d$.

Note that for $d=2.5 \AA$ the distance between ad-atom and chain atoms is only about $d=2.9 \AA$, hence smaller than the intra-chain nearest-neighbor distance of $d=3.0 \AA$. Nevertheless, $T(E)$ for $d=$ $2.5 \AA$ essentially is the same as the transmission for larger distances. For $d$ above $3.0 \AA$ the HOMO and its transmission peak remain unchanged; however, the LUMO shifts to lower energy with increasing $d$ (i.e., for decreasing $t_{\mathrm{s}}$ ).

Symmetry in the transport direction can be restored by placing the ad-atom below the central bond of a four-atom Li chain, see configuration $\mathrm{D}$. In this case the parent chain is insulating as we

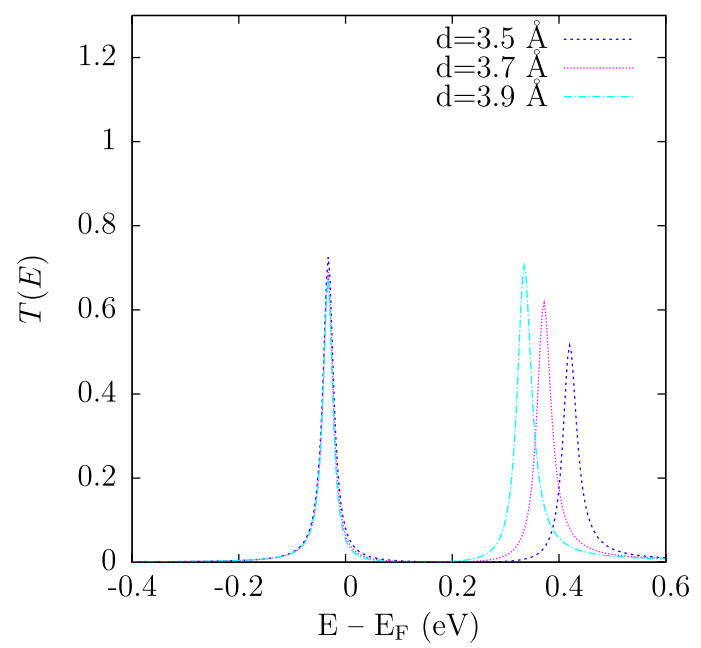

Figure $5 \mid$ Transmission coefficient for configuration $\mathrm{C}$ with strong $(\mathrm{a}=2.8 \AA$, left $)$ and weak $(\mathrm{a}=4.0 \AA$, right $)$ coupling to the leads. 

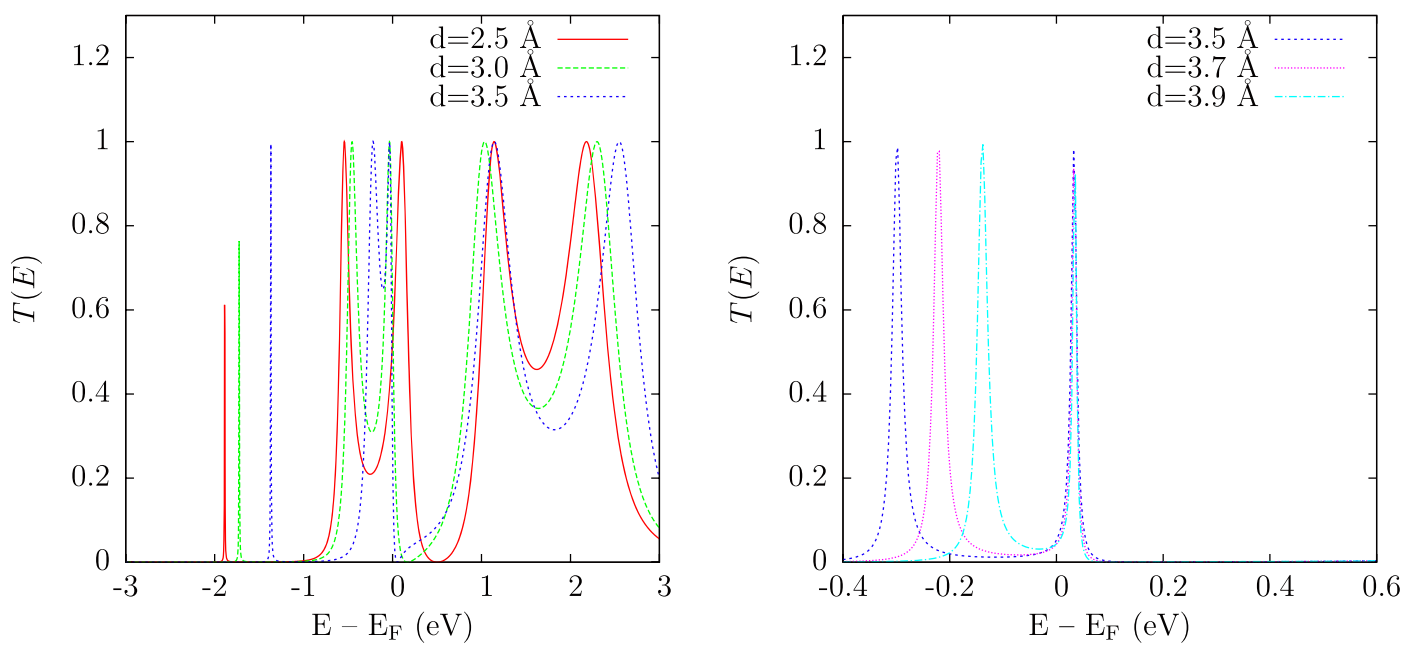

Figure $6 \mid$ Transmission coefficient for configuration D with strong $(a=2.8 \AA$, left $)$ and weak $(a=4.0 \AA$, right $)$ coupling to the leads.

have $\varepsilon_{1,2,3,4}=( \pm 1 / 2 \pm \sqrt{5} / 2) t_{\mathrm{par}}$. Including the impurity yields $\varepsilon_{1,4}=-t_{\text {par }} / 2 \pm \sqrt{5 t_{\text {par }}^{2}+8 t_{\mathrm{s}}^{2}} / 2, \varepsilon_{2,5}=t_{\mathrm{par}} / 2 \pm \sqrt{5} t_{\mathrm{par}} / 2$, and $\varepsilon_{3}$ $=0$. Thus, the ad-atom again switches the electronic state of the chain, but now from insulating to metallic. If we couple the central chain to the leads, $\varepsilon_{3}$ is shifted to higher energy, due to charge transfer, see Fig. 6. For $d>3.0 \AA$ the energy of the LUMO and its transmission peak remain essentially unaltered, but the distance to the HOMO decreases as $t_{\mathrm{s}}$ decreases, similar to the behavior of configuration $\mathrm{C}$.

We next study the influence of an impurity different from the atoms in the central region, comparing ad-atom and intra-chain configurations. As a prototypical example with high electronegativity we choose a fluor atom, which we place either adjacent to or incorporated into the Li chain. In the former case we expect, for an $\mathrm{F}$ atom attached to a single chain atom, that one Li electron will be trapped in the Li-F cluster, no longer contributing to transport. According to the left hand side of Fig. 7, the transmission spectrum of a seven-atom $\mathrm{Li}$ chain with $\mathrm{F}$ ad-atom in the middle ( $d=2 \AA$ away from the chain) consists of six peaks, confirming the above picture of one trapped electron. We note that the chain remains metallic despite the impurity. This can be understood by studying an equivalent system with a six-atom $\mathrm{Li}$ chain and a weak link at the center. To model the weak

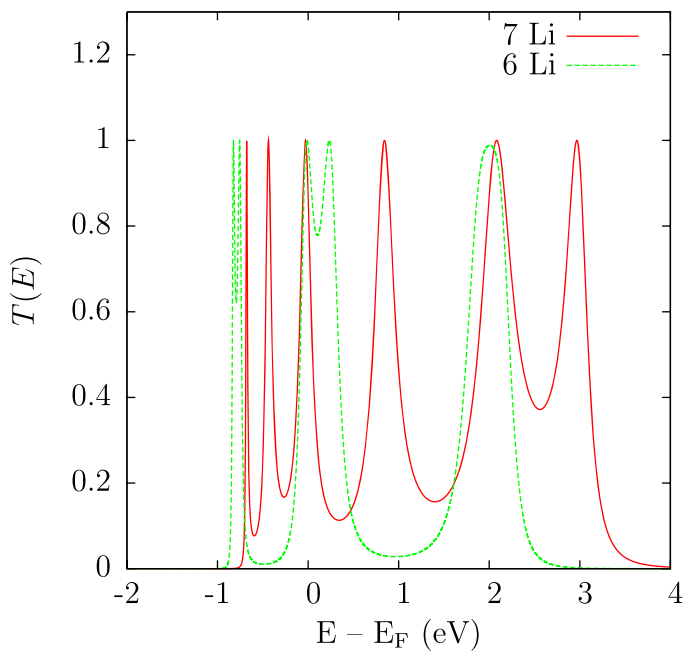

link, we increase the distance between the two central Li atoms to $5 \AA$. Effectively, we obtain the transmission spectrum of two three-atom Li chains, see the dashed curve on the left hand side of Fig. 7, i.e., metallic behavior and a high transmission at $E_{F}$.

The situation is different when the electronegative atom is placed within the chain, as shown on the right hand side of Fig. 7 for a Li-LiF-Li configuration with a Li-F distance of $1.5 \AA$. We find that the F atom forms a site with large attractive potential, since one transmission peak appears at very low energy (at about $-8.2 \mathrm{eV}$ ). We note that the resonance level at $E_{F}$ does not attain a transmission of 1 . While an $\mathrm{F}$ ad-atom forms a neutral cluster with the adjacent $\mathrm{Li}$ atom, an intra-chain $\mathrm{F}$ atom couples to both Li neighbors and acts as strong attractive potential.

Before summarizing our findings, we wish to comment on the following issues, in order to clearly put our work and our results into the proper context. First, our study is an extension of the paper by $\mathrm{Ke}$ et al. ${ }^{18}$ who studied the same type of toy model, concentrating on the energy-gap and self-interaction errors. To this end, six functionals with different exchange-correlation potentials were compared, with the conclusion that the optimized effective potential approach is a promising direction for improving DFT calculations. This agrees with the findings of Schenk et al. ${ }^{10}$ for an even simpler model system, namely a chain of spinless lattice fermions with nearest-neighbor

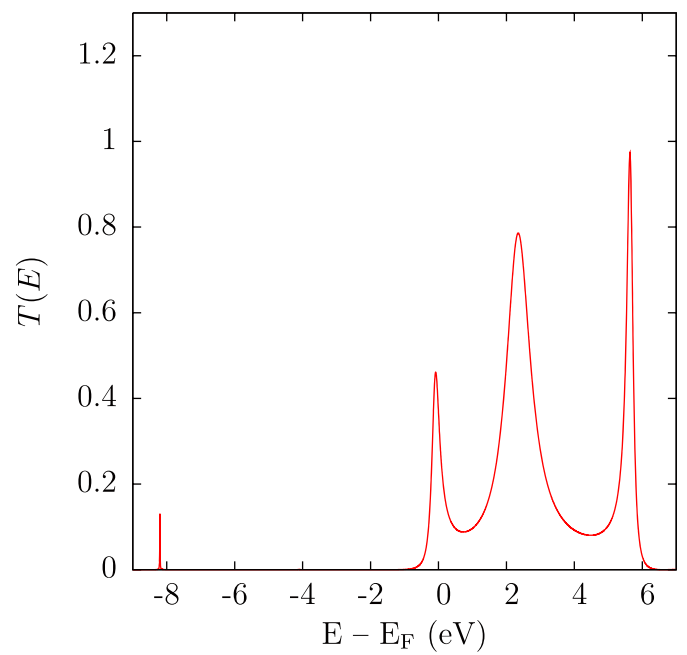

Figure 7 Left: Transmission coefficient for a seven-atom Li chain with an F ad-atom attached directly to the central Li atom, and for a six-atom Li chain with weak link at the center. Right: Transmission coefficient for a Li chain with intra-chain F impurity. 
interaction, provided the interaction is smaller than the bandwidth. For intermediate and large interaction, a combination of DFT with exact diagonalization within the scattering region is found to give reliable results for the linear conductance. We note that in relation to DFT-based transport calculations, fundamental open questions remain ${ }^{23}$.

In contrast to Ref. 18, the present work is based on the localdensity approximation, in view of its computational simplicity, hence we cannot expect that all details of our results are quantitatively correct. On the other hand, we present a systematic study of impurity effects, clarifying trends and possible szenarios. As discussed above, our findings can be interpreted well within a tight-binding description, and are hence physically transparent. Thus, even though Li (and $\mathrm{H}$ ) are known not to form chains, we expect that our results are not just a specific feature of our model, but are actually more generally valid.

\section{Discussion}

We have investigated the influence of impurities on the transmission of metallic and insulating monovalent Li chains. We have demonstrated that an impurity connected to a single atom of the chain leads to a splitting of the zero-energy peak, causing a strong dependence of the conductance on structural details of the scatterer. An impurity connected to two atoms of the chain with equal distance, on the other hand, switches the system from insulating to metallic behavior, or vice versa. The transmission coefficient, however, in both cases shows a minimum at $E_{F}$ as a consequence of the charge transfer with the leads. Charge transfer with the leads prohibits a prediction of details of the transmission spectrum by tight-binding models of the scatterer. Electronegative ad-atoms localize electrons in the chain but do not decouple it, whereas electronegative impurities within the chain result in low-lying transmitting levels and, thus, shift the spectrum to higher energy. Our results demonstrate that switches based on mono-atomic chains show a high variability of possible modifications that can be used to tailor the switching behavior.

\section{Methods}

We calculate the transmission through nanowires using a combination of scattering theory and density functional theor ${ }^{24,25}$, based on the SMEAGOL and SIESTA ${ }^{26}$ codes. A single zeta basis set and the local density approximation for the exchangecorrelation potential are used. In order to model the experimental situation (without gate voltage), metallic leads are connected to a central scattering region. In our case the leads are $\mathrm{H}$ chains, see Fig. 1. In the Landauer-Büttiker formalism, the selfenergies of the left $(\mathrm{L})$ and right $(\mathrm{R})$ leads are calculated first. The screening within the metallic leads ensures that effects of the contact region decay within a few nanometers. Since the leads are connected to the central scattering region (molecule, nanocontact, or interface), an effective description of the central region (C) emerges which includes the properties of the leads. In linear response, the transmission coefficient is given by the retarded Green's function $G_{C}$ of the central region and the lead selfenergies $\Sigma_{L / R}$. With $\Gamma_{L / R}=\mathrm{i}\left[\Sigma_{L / R}(E)-\sum_{L / R}^{\dagger}(E)\right]$ we have ${ }^{27}$

$$
T(E, V=0)=\operatorname{Tr}\left[\Gamma_{L} G_{C}^{\dagger} \Gamma_{R} G_{C}\right] .
$$

The conductance is given by $G=\frac{2 e^{2}}{h} T\left(E_{F}\right)$, where the factor 2 accounts for the spin degeneracy ${ }^{15}$.

1. Scheer, E. et al. The signature of chemical valence in the electrical conduction through a single-atom contact. Nature 394, 154-157 (1998).

2. Hansen, K. et al. Current-voltage curves of gold quantum point contacts revisited. Appl. Phys. Lett. 77, 708-710 (2000).

3. van Ruitenbeek, J., Scheer, E. \& Weber, H. Introducing Molecular Electronics 253-274 (Springer, Berlin, 2005).
4. Rodrigues, V., Fuhrer, T. \& Ugarte, D. Signature of atomic structure in the quantum conductance of gold nanowires. Phys. Rev. Lett. 85, 4124-4127 (2000).

5. Nilius, N., Wallis, T. M. \& Ho, W. Development of one-dimensional band structure in artificial gold chains. Science 297, 1853-1856 (2002).

6. Kane, C. L. \& Fisher, M. P. A. Transport in a one-channel Luttinger liquid. Phys. Rev. Lett. 68, 1220-1223 (1992)

7. Safi, I. \& Schulz, H. J. Interacting electrons with spin in a one-dimensional dirty wire connected to leads. Phys. Rev. B 59, 3040-3059 (1999).

8. Cuniberti, G., Sassetti, M. \& and Kramer, B. Ac conductance of a quantum wire with electron-electron interactions. Phys. Rev. B 57, 1515-1526 (1998).

9. Schmitteckert, P. \& Evers, F. Exact ground state density-functional theory for impurity models coupled to external reservoirs and transport calculations. Phys. Rev. Lett. 100, 086401 (2008).

10. Schenk, S., Schwab, P., Dzierzawa, M. \& Eckern, U. Density functional theory for a model quantum dot: Beyond the local-density approximation. Phys. Rev. B 83, 115128 (2011).

11. Runge, E. \& Gross, E. K. U. Density-functional theory for time-dependent systems. Phys. Rev. Lett. 52, 997-1000 (1984).

12. Kurth, S., Stefanucci, G., Almbladh, C.-O., Rubio, A. \& Gross, E. K. U. Timedependent quantum transport: A practical scheme using density functional theory. Phys. Rev. B 72, 035308 (2005).

13. Chico, L. \& Falicov, L. M. Electron scattering at interfaces: A tight-binding approach. Phys. Rev. B 52, 6640-6646 (1995).

14. Cuevas, J. C., Yeyati, A. L. \& Martín-Rodero, A. Microscopic origin of conducting channels in metallic atomic-size contacts. Phys. Rev. Lett. 80, 1066-1069 (1998).

15. Büttiker, M., Imry, Y., Landauer, R. \& Pinhas, S. Generalized many-channel conductance formula with application to small rings. Phys. Rev. B 31, 6207-6215 (1985).

16. Terabe, K., Hasegawa, T., Nakayama, T. \& Aono, M. Quantized conductance atomic switch. Nature 433, 47-50 (2005).

17. Hino, T. et al. Atomic switches: atomic-movement-controlled nanodevices for new types of computing. Sci. Technol. Adv. Mater. 12, 013003 (2011).

18. Ke, S. H., Baranger, H. U. \& Yang, W. Role of the exchange-correlation potential in ab initio electron transport calculations. J. Chem. Phys. 126, 201102 (2007).

19. Chakrabarti, A. Fano resonance in discrete lattice models: Controlling lineshapes with impurities. Phys. Lett. A 366, 507-512 (2007).

20. Solomon, G. C. et al. Understanding quantum interference in coherent molecular conduction. J. Chem. Phys. 129, 051701 (2008).

21. Ke, S. H., Yang, W. \& Baranger, H. U. Quantum-interference-controlled molecular electronics. Nano Lett. 8, 3257-3261 (2008).

22. Bergfield, J. P. \& Stafford, C. A. Many-body theory of electronic transport in single-molecule heterojunctions. Phys. Rev. B 79, 245125 (2009).

23. Schmitteckert, P., Dzierzawa, M. \& Schwab, P. Exact time-dependent density functional theory for impurity models. Phys. Chem. Chem. Phys. 15, 5477-5481 (2013).

24. Rocha, A. R. et al. Towards molecular spintronics. Nature Mater. 4, 335-339 (2005).

25. Rocha, A. R. et al. Spin and molecular electronics in atomically generated orbital landscapes. Phys. Rev. B 73, 085414 (2006).

26. Soler, J. M. et al. The SIESTA method for ab initio order-N materials simulation. J. Phys.: Condens. Matter 14, 2745 (2002).

27. Meir, Y. \& Wingreen, N. S. Landauer formula for the current through an interacting electron region. Phys. Rev. Lett. 68, 2512-2515 (1992).

\section{Acknowledgments}

We thank S. Schenk and P. Schwab for discussions, and acknowledge financial support from the Deutsche Forschungsgemeinschaft (through TRR 80).

\section{Author contributions}

T.W., B.A. and C.S. performed the calculations. U.E., C.S. and U.S. wrote the manuscript.

\section{Additional information}

Competing financial interests: The authors declare no competing financial interests.

How to cite this article: Wunderlich, T., Akgenc, B., Eckern, U., Schuster, C. \& Schwingenschlögl, U. Modified Li chains as atomic switches. Sci. Rep. 3, 2605; DOI:10.1038/ srep02605 (2013)

cc) (i) $\Theta$ This work is licensed under a Creative Commons Attribution-

BY ${ }_{\mathrm{NC}} \mathrm{ND}$ NonCommercial-NoDerivs 3.0 Unported license. To view a copy of this license, visit http://creativecommons.org/licenses/by-nc-nd/3.0 